\title{
En bloc aortic root resection and Commando procedure for aortomitral bioprosthetic endocarditis
}

\author{
Gaby Aphram, MD, Jama Jahanyar, MD, PhD, Laurent de Kerchove, MD, PhD, and \\ Gebrine El Khoury, MD, PhD, Brussels, Belgium
}

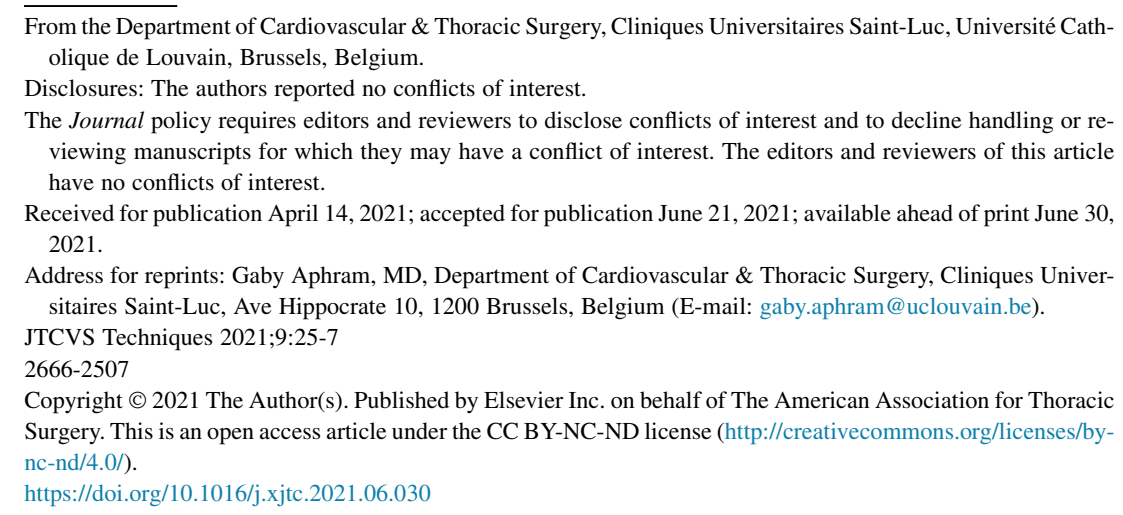

Video clip is available online.

Early aortomitral bioprosthetic degeneration with acute endocarditis represents a surgical challenge because it requires thoughtful preoperative evaluation and anticipation of a complex and difficult reoperation. The aortomitral continuity and fibrous skeleton is often completely destroyed, and hence mandates a double valve replacement with recreation of a new aortomitral continuity, with a pericardial patch for instance. This technique was originally described by Tirone David, and was named Commando procedure by the Cleveland Clinic group. ${ }^{1}$

Approaching the aortic root in the setting of redo surgeries is a challenge in and of itself, and herein we describe our technique for en bloc aortic root resection, followed by a Commando procedure.

\section{CLINICAL SCENARIO}

A 60-year-old woman with a past medical history significant for rheumatic heart disease, underwent a mitral and aortic valve replacement 2 years prior at an outside institution, with a 31-mm Hancock II (Medtronic, Minneapolis, Minn) and 21-mm Avalus (Medtronic) bioprosthesis, respectively. After experiencing acute limb ischemia that required emergency embolectomy, she was diagnosed with early and significant aortic valve degeneration and

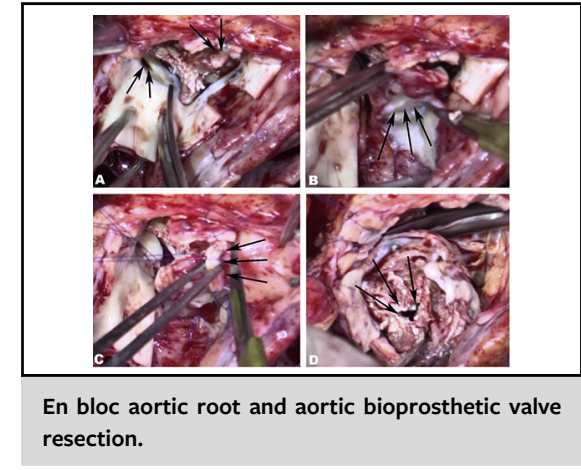

\section{CENTRAL MESSAGE \\ En bloc aortic root resection fa- cilitates redo root surgeries, and in acute aortic valve endocarditis it ensures resection of all in- fected tissues.}

See Commentary on page 28.

double-valve endocarditis with Streptococcus vestibularis. She was transferred to us for double valve replacement.

\section{SURGICAL TECHNIQUE}

The surgical technique is detailed in Video 1. After redo sternotomy and careful adhesiolysis, cardiopulmonary bypass was established via central aortic and bicaval venous

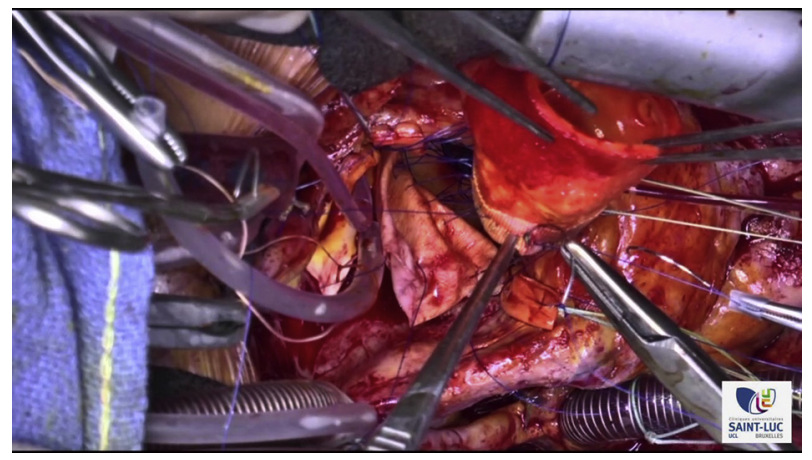

VIDEO 1. Double-valve replacement with redo en-bloc aortic root resection and Commando procedure, for degenerative bioprosthetic aortic valve disease and left-sided double-valve endocarditis. Video available at: https://www.jtcvs.org/article/S2666-2507(21)00429-6/fulltext. 

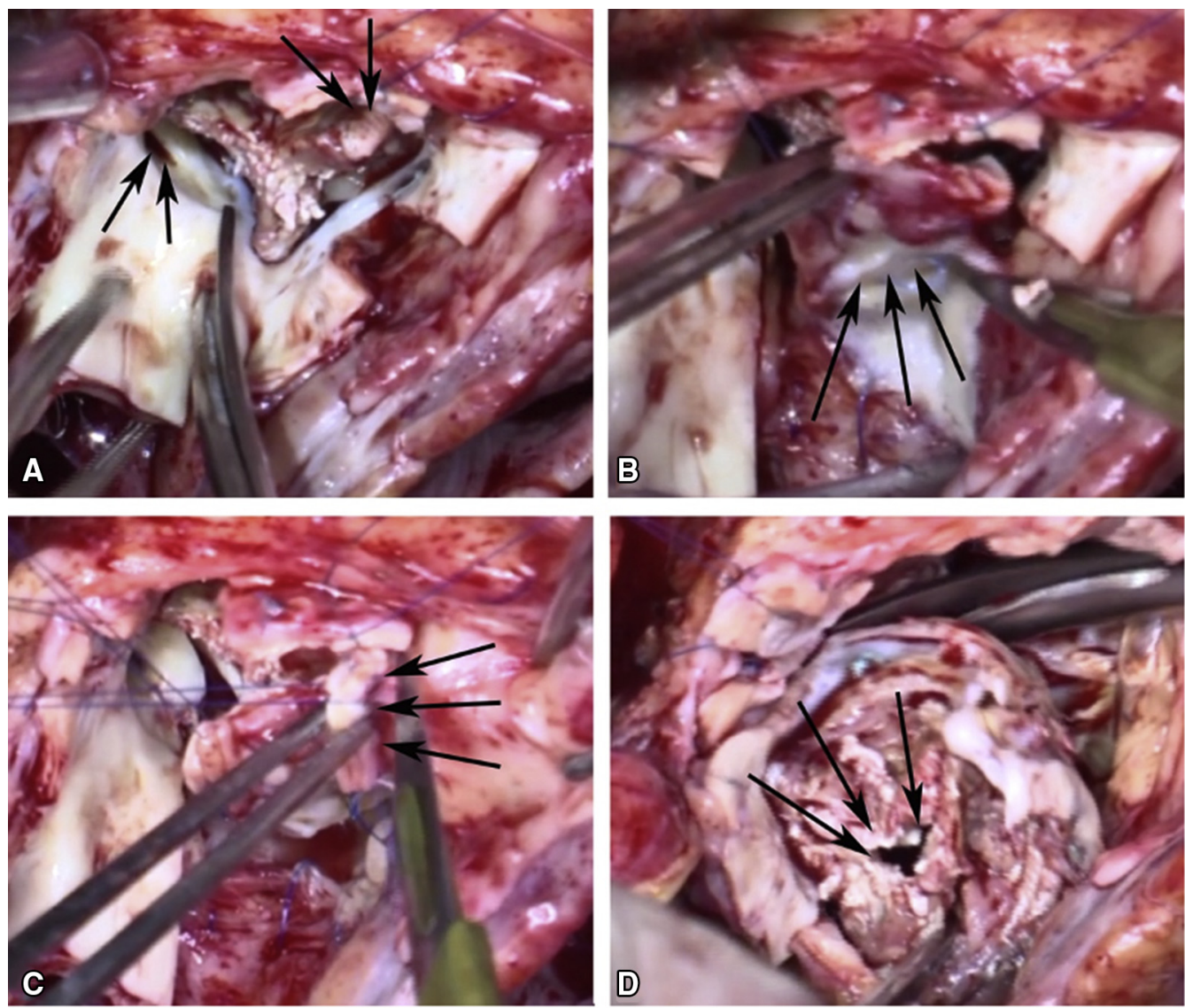

FIGURE 1. A, En bloc aortic root resection. First and most important step is to mobilize and protect the coronary arteries (arrows depict the right coronary artery anteriorly and the left coronary artery posteriorly). B, The root resection is started at the non/left coronary commissure and is carried out counterclockwise. The dissection is carried out towards the left ventricular outflow tract, outside of the commissure and includes the commissure in the specimen (arrows depict the non/left coronary commissure). C, Dissection is continued behind the non/right coronary commissure (arrows depict the non/right commissure). $\mathrm{D}$, Dissection is completed with the scissors. The aortic valve prosthesis is removed in toto with the aortic root tissues (arrows depict the severely stenotic bioprosthetic aortic valve).

cannulation. Following cardiac arrest with intermittent normothermic antegrade blood cardioplegia, the ascending aorta was transected $1.5 \mathrm{~cm}$ above the sinotubular junction. An abscess was identified in the aortomitral curtain. The right and left coronary buttons were mobilized first, which is the initial step for the en bloc root resection. The remainder of the aortic root, including the aortic bioprosthesis and parts of the aortomitral curtain, were then resected en bloc, which opened the left atrium as well (Figure 1). The mitral bioprosthesis was then removed through the aortic root. The infected tissues were aggressively debrided, and the now-created common left-sided chamber was thoroughly irrigated with normal saline. A 31-mm Magna-Ease (Edwards Lifesciences, Irvine, Calif) mitral bioprosthesis, which was briefly soaked in a rifampin-based solution, was anchored to the posterior mitral annulus with 2.0 Ti-Cron (Medtronic, Minneapolis, $\mathrm{MN}$ ) horizontal mattress sutures from the posteromedial to the anterolateral trigone. An equine pericardial strip was utilized in lieu of pledgeted valve sutures to reduce the amount of pledgets in this endocarditis case. Once the valve was secured to the posterior mitral annulus, another larger equine pericardial patch, which had been folded onto itself, was sewn in a running fashion to the anterior bioprosthetic valve annulus with a 4.0 Prolene (Ethicon Inc, Cornelia, Ga) suture, form trigone to trigone. This served the purpose of re-establishing the aortomitral continuity and to provided coverage to close the roof of the left atrium. With this, the left ventricular inflow and outflow tracts were re-established. Hence, after the patch was sewn to the mitral valve prosthesis, the posterior wing of the double patch closed the roof because it was sewn to the free edges of the left atrial dome. A 23-mm Freestyle (Medtronic) stentless bioprosthesis was then sutured with a 4.0 Prolene suture to the anterior wing of the double patch and the anterior aortic annulus in a running fashion, leaving roughly $1 \mathrm{~cm}$ of patch above the anterior mitral annulus for the aortomitral continuity. The Freestyle graft was sprayed with 
rifampin as well. A second hemostatic running suture was routinely performed between the Freestyle root and the surrounding tissues.

The left coronary button was then reimplanted in the usual fashion; in this case, the right coronary artery was severely scarred and could not be sufficiently mobilized for reimplantation. It was therefore ligated and bypassed with an autologous vein graft to the proximal right coronary artery. After the distal aortic anastomosis and proximal vein graft anastomosis were completed, the patient was weaned off cardiopulmonary bypass (Video 1). The postoperative transesophageal echocardiography showed normal function of both valves and the postoperative course of the patient was unremarkable and uneventful. We obtained written informed consent from the patient for publication of this case report. Institutional review board approval for this report was not required by our institution.

\section{DISCUSSION}

We routinely perform en bloc aortic root resections in redo aortic root replacements, and in cases of aortic valve endocarditis with possible root abscess. After mobilization of both coronary buttons, the aortic root with commissures and native or prosthetic valve is removed in toto. In this patient, our en bloc aortic root resection was combined with resection of an infected mitral valve bioprosthesis through the aortic root.

The benefits of the en bloc aortic root resection technique are numerous. We avoid contamination, such as in endocarditis cases with complete removal of all infected tissues of the aortic annulus, we avoid debris, and it immediately cuts to healthy tissues to which to sew.

In our experience, we have not encountered any major complications with our technique, such as membranous ventricular septal defects or need for permanent pacemakers. The most critical part of the en bloc resection is the mobilization and protection of the coronary arteries. The new aortic prosthesis is then sewn to the aortomitral curtain posteriorly (in this case the aortomitral curtain was recreated), and the interventricular septum anteriorly. Possible perforations in the muscular septum can be incorporated into the suture line, or in case of the membranous septum, can be closed primarily or with a small pericardial patch.

When the infection carries on below the level of the aortic annulus, then further debridement is needed, such as in this case where the mitral bioprosthesis with adjacent infected tissues had to be debrided as well. We aggressively remove all infected tissues, as well as all of the previously placed pledgets. We believe that the felt pledgets function as a bouillon de culture; that is, a culture medium for further bacterial growth. If injury to the posterior mitral annulus would occur with this radical debridement, we would also repair it during placement of the posterior valve sutures.

Double-valve endocarditis is a technically challenging clinical picture associated with significant morbidity and mortality. Prosthetic endocarditis, increasing age, preoperative shock, and diabetes mellitus were independent predictors of death from all causes in a study from Toronto. ${ }^{2}$ Radical debridement and reconstruction form the cornerstones in infective endocarditis. The surgical risk is greater in patients with active endocarditis. ${ }^{3}$

This procedure was performed at a center with decades of high-volume aortic root surgery experience. We illustrated a possible solution to a complex clinical scenario. Caution and good judgment should be exercised when approaching this pathology because the results may or may not be reproducible by less-experienced surgeons.

\section{References}

1. David TE, Kuo J, Armstrong S. Aortic and mitral valve replacement with reconstruction of the intervalvular fibrous body. J Thorac Cardiovasc Surg. 1997; 114: 766-71.

2. Sheikh AM, Elhenawy AM, Maganti M, Armstrong S, David TE, Feindel CM Outcomes of double valve surgery for active infective endocarditis. J Thorac Cardiovasc Surg. 2009;138:69-75.

3. Gillinov AM, Diaz R, Blackstone EH, Pettersson GB, Sabik JF, Lytle BW, et al Double valve endocarditis. Ann Thorac Surg. 2001;71:1874-9. 